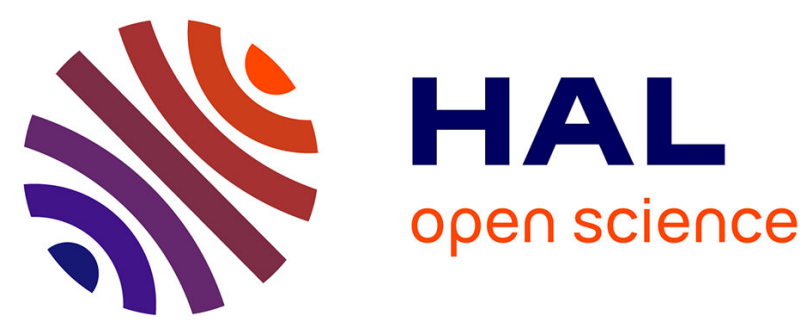

\title{
Les ostraca grecs et coptes d'Edfou. À propos d'une publication récente
}

\author{
Alain Delattre, Jean-Luc Fournet
}

\section{To cite this version:}

Alain Delattre, Jean-Luc Fournet. Les ostraca grecs et coptes d'Edfou. À propos d'une publication récente. Archiv für Papyrusforschung und verwandte Gebiete, 2011, 57, pp.79-98. hal-01597287

\section{HAL Id: hal-01597287 \\ https://hal.science/hal-01597287}

Submitted on 28 Sep 2017

HAL is a multi-disciplinary open access archive for the deposit and dissemination of scientific research documents, whether they are published or not. The documents may come from teaching and research institutions in France or abroad, or from public or private research centers.
L'archive ouverte pluridisciplinaire HAL, est destinée au dépôt et à la diffusion de documents scientifiques de niveau recherche, publiés ou non, émanant des établissements d'enseignement et de recherche français ou étrangers, des laboratoires publics ou privés. 


\title{
Les ostraca grecs et coptes d'Edfou. À propos d'une publication récente
}

\author{
Planche IV \\ Alain Delattre (Leiden) et Jean-Luc Fournet (Paris)*
}

\begin{abstract}
Corrections and reeditions of Greek, Coptic and Graeco-Coptic ostraca from Edfu (Egypt) recently published by S. Bacot, Ostraca grecs et coptes de Tell Edfou, Le Caire, 2009 (O.Edfou Copte)
\end{abstract}

Keywords: Edfu, ostraca, Menander's Sententiae, Psalms, New Testament

De 1937 à 1939 une mission archéologique franco-polonaise entreprit des fouilles sur le site d'Edfou. Une bonne partie de la documentation grecque fut publiée dans les rapports de fouilles ${ }^{1}$, tandis que le versant copte resta négligé ${ }^{2}$. Il a fallu attendre 2009 pour voir cette lacune enfin comblée grâce à la publication de Seÿna Bacot $^{3}$. L'ouvrage comprend l'édition de 145 ostraca, pour la plupart inédits ${ }^{4}$ : les

* La présente contribution est le fruit d'une collaboration entre les auteurs, qui avaient tous deux préparé indépendamment une liste de corrections. Les apports respectifs des auteurs sont indiqués par leurs initiales.

${ }^{1}$ B. Bruyère et al., Fouilles franco-polonaises. Rapports. I. Tell Edfou 1937, Le Caire, 1937; K. Michałowski et al., Fouilles franco-polonaises. Rapports. II. Tell Edfou 1938, 2 fasc., Le Caire, 1938-1939; K. Michałowski et al., Fouilles franco-polonaises. Rapports. III. Tell Edfou 1939, Le Caire, 1950.

${ }^{2}$ Cf. A. Boud'hors, «Réflexions préalables à la publication des textes coptes d'Edfou (papyrus et ostraca)», dans Tell-Edfou soixante ans après. Actes du colloque franco-polonais, Le Caire, 15 octobre 1996, Le Caire, 1999, p. 1-7.

${ }^{3}$ S. Bacot, Ostraca grecs et coptes de Tell Edfou, Bibliothèque d'études coptes 19, Le Caire, 2009. La matière est divisée en plusieurs chapitres: la vie religieuse à Edfou (1-35), le quotidien d'un intendant (36-65), hommes et femmes de la ville et de la campagne (66-128), documents inclassables (129-145).

${ }^{4}$ Les textes suivants avaient déjà été édités: 1 (S. Bacot, «Un calendrier liturgique d'Edfou sur ostracon, l'O.Edfou Copt. Lit. 1», dans A. Boud'hors, C. Louis, Études coptes X. Douzième journée d'études, Lyon, 19-21 mai 2005, Paris, 2008, p. 92-101); 40-42 (S. Bacot, «Quelques textes relatifs aux mesures de vin d'Edfou au VII siècle», dans A. Boud'hors, J. Gascou, D. Vaillancourt, Études coptes IX. Onzième journée d'études (Strasbourg, 12-14 juin 2003), Paris, 2006, p. 33-44); 39, 44, 45, 46, 47 (Ch. Heurtel, «Reçus coptes d'Edfou (Musée du Louvre et I.F.A.O.)», dans M. Rassart-Debergh, Études coptes V. Sixième Journée d'Études. Limoges 18-20 juin 1993 et Septième Journée d'Études. Neuchâtel 18-20 mai 1995, Paris-Louvain, 1998, p. 137-153); 107 (S. Bacot, «Le vin à Edfou», dans S. Marchand, A. Marangou, Amphores d'Égypte de la Basse 
pièces coptes sont les plus nombreuses, mais la part grecque est malgré tout substantielle5. Malheureusement, comme le dit l'auteur (p. VII), l'état de conservation des ostraca rend souvent difficiles la lecture et l'interprétation des documents; nous proposons ici quelques remarques et corrections destinées à faire progresser l'édition de ces ostraca, et de là nos connaissances sur la ville d'Edfou à la fin de la période byzantine et au début de l'époque arabe.

\section{O.Edfou Copte 3 [AD]}

L'ostracon conserve les restes d'une composition psalmique sur le thème de la pitié du Seigneur ${ }^{6}$. Au début de la 1. 16, la séquence єіт2нто еst sans doute la fin d'une citation partielle du Ps 89, 16 (NгXı MOGIT гнтоY NNGY()нPE), intercalée entre des extraits de Ps 89, 15 (1.15) et 89, 17 (1. 16).

À la dernière ligne (22), éditée NoY( $) d x \in \epsilon \ldots$. . . on peut reconnaître le Ps

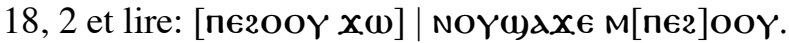

\section{O.Edfou Copte 10 [JLF]}

L'éditrice a bien noté que «certains mots évoquent Lc 24,1-2». Mais, plus qu'à des échos, on a affaire à une sorte de paraphrase de l'épisode des «femmes au

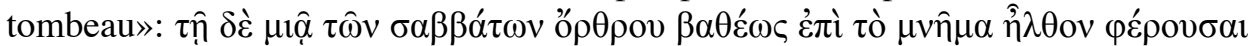

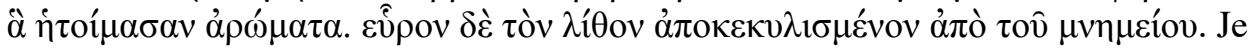
proposerais le texte suivant (le texte de droite est celui de l'édition):

$$
\text { éd. }
$$

1 ligne abîmée

po vv.....

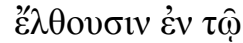

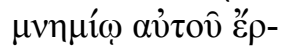

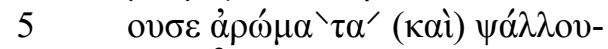

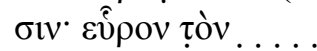

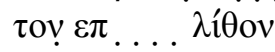

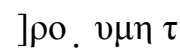

$\dot{\varepsilon} \lambda \theta 0 v \sigma \mathrm{v} v \dot{\varepsilon} v \tau \omega[$ $\mu \nu \eta \mu \mathrm{\omega} \alpha \mathrm{v} \tau \mathrm{O} \mathrm{v} \varepsilon$.

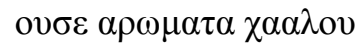

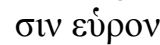
$\tau \mathrm{ov} \varepsilon \pi . \lambda \mathrm{l} \theta \mathrm{ov}$

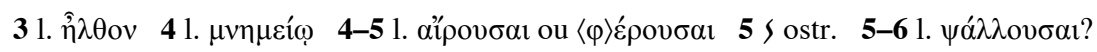

Époque à l'époque arabe, vol. 2, Le Caire, 2007, p. 713-720). Enfin, le texte 91 a été mentionné par J. Gascou (J. Gascou, «La table budgétaire d'Antaeopolis (P.Freer 08.45 c-d)», dans Hommes et richesses dans l'Empire byzantin. I: IV -VII siècle. Réalités byzantines, Paris, 1989, p. 279-313, en part. p. 296, n. 92 = Fiscalité et société en Égypte byzantine, Paris, 2008, p. 332, n. 92).

${ }^{5}$ Sur 145 documents, 93 sont coptes $(1-9,11-35,44-45,58,60,63-74,76-87,92-93,104$, $107-120$ et 122-145), 26 sont grecs $(10,48-54,59,75,88-91,94-103$ et 105-106) et 26 sont bilingues grec-copte $(36-43,46-47,55-57,61-62$ et 121). Une telle composition pourrait peutêtre faire préférer un sigle comme $O . E d f o u$ Ifao.

6 Ces compositions, parfois thématiques, sont définies comme «concordances»; il semble préférable de garder ce terme pour les vraies concordances, comme O.Mon. Epiph. 16 AC. 
«[...] Elles allèrent à son tombeau, apportant des aromates et psalmodiant (litt. elles psalmodient). Elles trouvèrent $[. .$.$] la pierre [...].»$

Le texte suit de près celui de l'Évangile à ceci près qu'il fait psalmodier les femmes pendant le trajet, détail absent de la tradition. Il le simplifie en l'adaptant, avec des fautes, à la langue courante de l'époque (1. 3-4, $\dot{\varepsilon} v+$ datif pour $\dot{\varepsilon} \pi \dot{\imath}+$ accusatif).

3 '̌ $\lambda \theta$ ovorv: on pourrait croire qu'on a un participe au datif ( $\dot{\varepsilon} \lambda \theta 0 \hat{\sigma} \sigma)$, comme

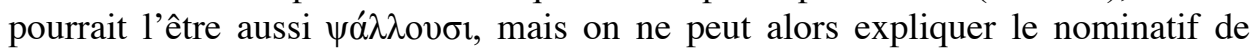

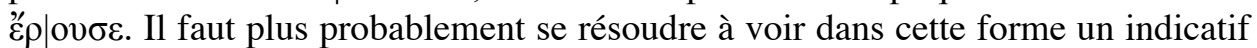
aoriste (sans augment et avec une désinence de présent!) ou un subjonctif aoriste (avec une désinence d'indicatif, cf. Mandilaras, Verb, § 543) dépendant d'une conjonction maintenant perdue avec les lignes précédentes (cf. Mc 16, 1: ク่ ó $\rho \alpha-$

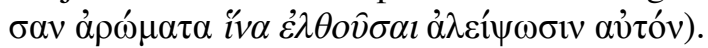

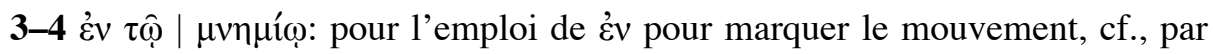
exemple, A.N. Jannaris, An Historical Greek Grammar Chiefly of the Attic Dialect, Londres 1897, § 1565; F. Blass et A. Debrunner, A Greek Grammar of the New Testament and Other Early Chritian Literature, révisée par R.W. Funk, Chicago 1961, § 218. Le diminutif $\mu v \eta \mu \varepsilon i ̂ v$, à la place de $\mu v \hat{\eta} \mu \alpha$, est employé par Luc à la fin de 24, 2.

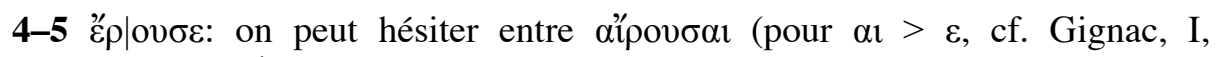

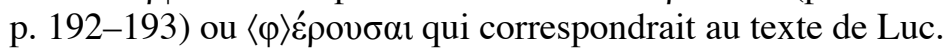

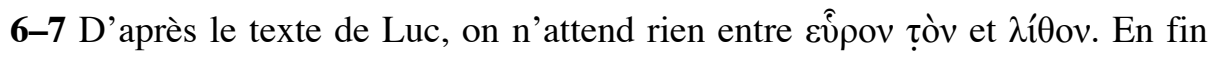

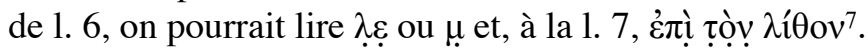

\section{O.Edfou Copte 11 [AD-JLF]}

L'éditrice voyait dans cet ostracon un «texte pieux» copte. On doit en fait y

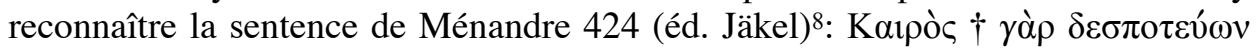

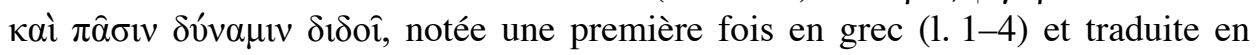
copte (1. 4-7), très probablement dans un contexte scolaire. Le texte de la sentence a été transmis par une partie de la tradition manuscrite et on la trouve aussi dans

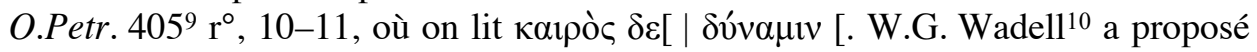

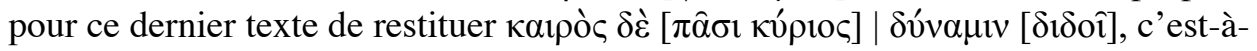
dire une des reconstitutions proposées par J.Fr. Boissonade pour rendre métrique

\footnotetext{
7 Je dois ces informations à Florence Lemaire, qui a bien voulu contrôler cet ostracon à ma demande. Je l'en remercie.

${ }^{8}$ Voir aussi C. Pernigotti, Menandri Sententiae, Florence, 2008, p. 424 (apparat plus précis).

$9=$ LDAB 2450, Mertens-Pack ${ }^{3} 1587$.

10 «Some Literary Papyri from Oxyrhynchus», Études de papyrologie 1, 1932, p. 18.
} 
le texte des manuscrits ${ }^{11}$. L'ostracon d'Edfou apporte un nouveau témoin de cette sentence, proche d'O.Petr. 405, qu'il convient désormais de restituer à partir de notre texte ${ }^{12}$.

La partie grecque de l'ostracon est corrompue, mais on peut la reconstituer à partir de la traduction copte: $\mathbf{\epsilon T} \mid \mathbf{O}$ NXX€ıIC, «qui est maître», doit correspondre au

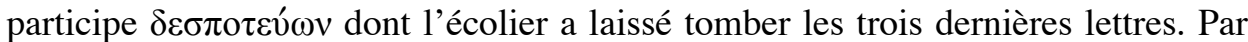

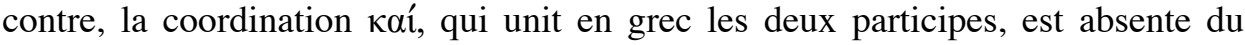
copte, où OYNTAч fait office de verbe principal.

La sentence conservée dans l'ostracon commence de la même manière que celle de la tradition manuscrite, mais ne reprend pas la particule $\gamma \alpha$ ó $\rho$ (de même

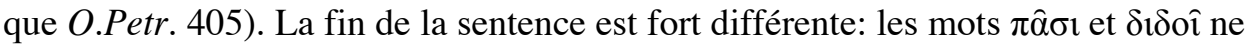
se retrouvent pas dans notre texte. On notera que la place de $\pi \hat{\alpha} \sigma \mathrm{t}$ varie selon les manuscrits et que $\delta$ ioô̂ est une correction moderne: les témoins médiévaux

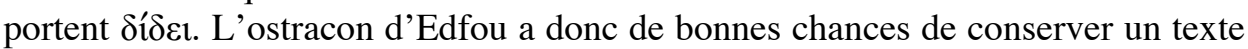
plus proche de l'original, même si son caractère amétrique indique que le texte est corrompu.

Il vient s'ajouter à P.Rain. Unterricht Kopt. 268 (prov. inconnue), autre ostracon scolaire contenant une sentence de Ménandre en grec puis en copte, de même époque (VII ${ }^{\mathrm{e}}$ s.), ainsi qu'aux P.Rain. Unterricht Kopt. 269 I (prov. inconnue, $\mathrm{VI}^{\mathrm{e}} / \mathrm{VII}^{\mathrm{e}} \mathrm{s}$.) et II (prov. inconnue, $\mathrm{V}^{\mathrm{e}} / \mathrm{VI}^{\mathrm{e}} \mathrm{s}$.), livres d'école consacrés aux Sentences de Ménandre en version bilingue gréco-copte ${ }^{13}$.

+ Kalpòৎ $\delta \varepsilon[\sigma-]$

$\pi \mathrm{o} \varepsilon<\dot{v} \omega v>\kappa \alpha i ̀$ \{ $\delta v$.

$\alpha 1\} \delta v^{\prime} \alpha \mu \mathrm{u} v{ }^{\prime} \chi-$

$\omega V$ กKגIPOC 6 T-

5 O NXOGIC OYN-

TAY NOYGO-

M +

1 kalpoc xe ed. 2 note kal aY. ed. 3 alaYNamine + ed. 6-7 nOYO|N ed.

«(grec) L'occasion, qui est souveraine et qui a la force. (copte) L'occasion, qui est souveraine, a la force.»

3-4 $\{\delta v . \mid \alpha 1\}$ : lire probablement $\delta v v \alpha \mu$, la dernière lettre n'ayant pas été terminée et se réduisant à son premier élément vertical. La raison pour laquelle

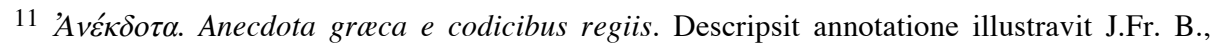
vol. 1, Paris, 1829, p. 156, n. 4.

${ }^{12}$ Il n'y a donc pas lieu de douter qu'O.Petr. soit un témoin de cette sentence comme semble le faire C. Pernigotti (en faisant suivre la référence à cet ostracon d'un ?).

13 Cf. D. Hagedorn et M. Weber, «Die griechisch-koptische Rezension der Menandersentenzen», ZPE 3, 1968, p. 15-50. 
l'écolier aurait interrompu cette séquence avant de la réécrire complètement nous échappe.

\section{O.Edfou Copte 16 [AD]}

Il faut reconnaître à la dernière ligne conservée une citation de Mt 2, 16, 1. La première ligne, qui commence par la séquence $n \times \omega \mathrm{M}$, pourrait être le début de

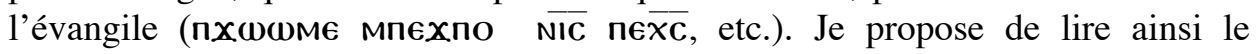
document:

$n^{\prime} \mathbf{x}^{\prime} \omega \mathrm{m}$ traces

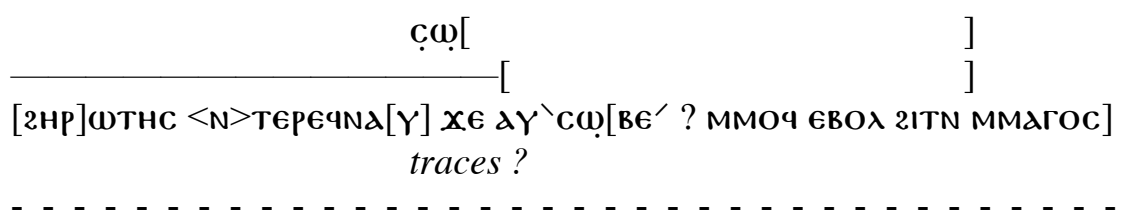

«Le livre (de la génération de Jésus, le Christ ?)... Alors Hérode, voyant qu'il avait été joué (par les mages, se mit dans une grande colère, et il envoya tuer tous les enfants de deux ans et au-dessous qui étaient à Bethléhem et dans tout son territoire...).»

\section{O.Edfou Copte 19 [AD]}

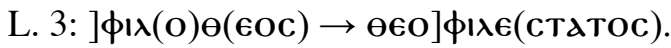

L. 7: пEN!IIT $\rightarrow$ nENEIWT.

\section{O.Edfou Copte 23 [AD]}

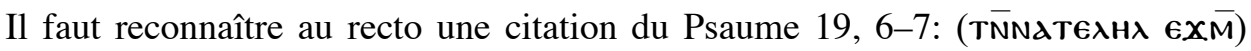

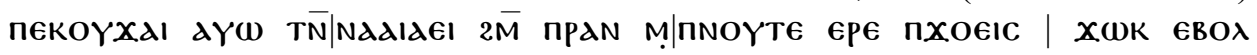

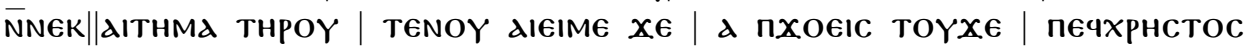
| єчєсСтм єРОк..

On lit dans la partie inférieure du recto, à $180^{\circ}$ par rapport au texte principal, deux lignes dénuées de sens, sans doute des exercices d'écriture; au verso, il y a le début d'une lettre. La nature des textes et l'écriture malhabile suggèrent une fonction «scolaire».

\section{O.Edfou Copte 33 [AD]}

À la dernière ligne (1.7), il faut lire un montant $\alpha \sigma \xi \varepsilon \mu\left(o^{\prime} \alpha \alpha\right)$, plutôt qu'une date (KÇ $\left.\in \Delta \mathrm{M}^{\prime}\right)$. Il s'agirait donc de 1.265 xestes d'huile, chiffre qui était apparemment écrit en copte et en toutes lettres à la ligne précédente, où l'on distingue .... C СеTH $\bar{N} z \in(\mathbf{C T H C}) \overline{\mathrm{N}} \mathrm{N} \in 2$, «... soixante-cinq xestes d'huile». Après le montant exprimé en grec, on voit un deuxième $\mu$ pourvu d'une marque d'abréviation, sans doute le début d'une date: $\mu(\eta v i ́)$, «au mois de». 


\section{O.Edfou Copte 36-65 [JLF]}

Ces ostraca sont rassemblés dans une subdivision intitulée «Le quotidien d'un intendant», l'éditrice identifiant le Sevêros qui apparaît dans la souscription de plusieurs de ces textes avec un homonyme dans lequel R. Rémondon voit «une sorte d'intendant de Papas» (P.Apoll. 63, 28; 65, 7; 8 et 101, 2). On notera, avec R. Rémondon (P.Apoll. 54, 4 n.), que ce nom est très répandu dans la documentation apollonopolite et qu'il est porté par divers fonctionnaires (un chartoularios, un apokrisarios). Par ailleurs, Sevêros n'est pas le seul à signer les reçus qui constituent ce sous-ensemble (comprenant aussi des comptes anonymes): on trouve un Silbanos (O.Edfou Copte 46, 47), un Mênas (O.Edfou Copte 53), un Matthaios (?) (O.Edfou Copte 54), un Andreas (O.Edfou Copte 55). De plus, le Sevêros (ou un des Sevêros) signant ces reçus apparaît en O.Edfou Copte 42, 4

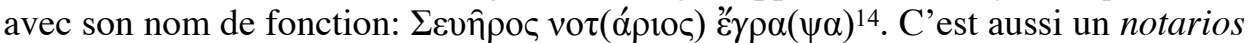
qui signe $O . E d f o u$ Copte 56. Le notarios est un secrétaire-tachygraphe (et non un notaire) attaché à un fonctionnaire; on ne s'attend pas à ce qu'un intendant de domaine soit ainsi désigné. Il est donc préférable d'interpréter les reçus compris dans O.Edfou Copte 36-65 comme des quittances d'impôt en nature signées par divers fonctionnaires de la pagarchie. Leur visa tantôt avec $\sigma \tau o \iota x \varepsilon \hat{\varepsilon}$, tantôt avec है $\gamma \rho \alpha(\psi \alpha)$ est d'ailleurs typique des documents fiscaux. On doit donc renoncer à voir dans cette série de textes (par ailleurs disparate) des témoignages de la gestion des propriétés privées de Papas.

\section{O.Edfou Copte 36-40: les reçus signés par Sevêros [AD]}

Aux cinq reçus signés de la main de Sevêros, on peut ajouter un document supplémentaire, publié en 1960 par St. Jakobielski ${ }^{15}$. Son formulaire est très proche de celui des textes 39-41 et les ostraca de l'IFAO permettent d'éclairer l'édition originale et inversement; je propose ici une réédition du document (planche IV) ${ }^{16}$.

O.Vars. Inv. 139901

+ 2ITOOTK NTOK IW2ANNHC

EIC C)OMNTE NBAICOOMNT $\overline{\mathrm{NHP}}$

NTE חME2CNAY NEIOOM NTA2WP AYEI

ETOOT NTEI2E ETKIMA $(\tau \rho) \pi \lambda(\hat{\alpha}) \alpha$ ME $\omega$ -

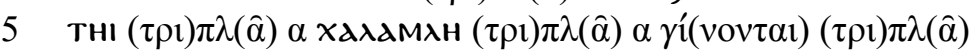

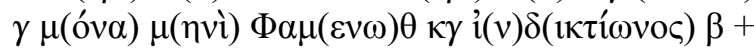

\footnotetext{
14 Voir ci-dessous

15 T. Andrzejewski, St. Jakobielski et S. Strelcyn, Katalog rekopisow orientalnych ze zbiorow polskich, Katalog rekopisow egipskich, koptyskich i etiopskich, Varsovie, 1960, en part. p. 25-42: St. Jakobielski, Rekopisy koptyjskie, III. 10 (p. 36-37).

16 Je remercie le Musée National de Varsovie et en particulier Mikołaj Machowski de m'avoir procuré une photographie de l'ostracon.
} 


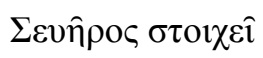

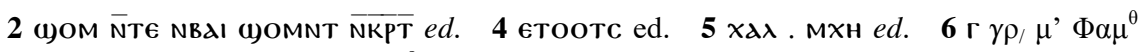

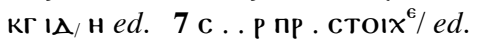

«Par tes soins à toi, Jôhannês, voici que trois baishomnt de vin de la deuxième cuvée de Tahôr me sont parvenus de cette manière: pour Tkima, 1 triploun; (pour) Mechtêi, 1 triploun; (pour) Chalamlê, 1 triploun. Total: 3 tripla seulement. Au mois de Phamenôth, le 23, $2^{\mathrm{e}}$ année de l'indiction. Sevêros marque son accord.»

Les séquences des lieux bénéficiaires de vin; ces trois toponymes ne sont pas attestés par ailleurs.

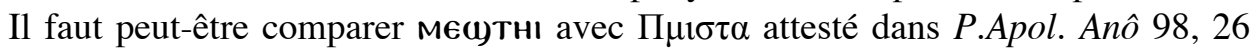

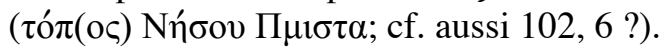

\section{O.Edfou Copte 36 [AD]}

L. 3: $\dot{\alpha} \theta \hat{v} \rho \kappa \alpha$ iv $\delta(1 \kappa \tau i ́ \omega v o \varsigma) \rightarrow{ }^{\circledR} A \theta v(\rho) \kappa \delta$ iv $\delta(1 \kappa \tau i ́ \omega v o \varsigma) \gamma$.

\section{O.Edfou Copte 36-43 et 55 [JLF]}

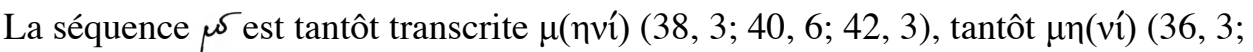
$37,3 ; 43,5)$, tantôt $\mu(\eta) v(\hat{i})(39,6)$. Il s'agit en fait d'un $\mu$ dont la fin est ligaturée à une marque d'abréviation. Seule la première transcription est correcte. On ne confondra pas cette séquence avec $\mathfrak{w}$ (O.Edfou Copte 55, 5): loin d'en être une variante (l'éd. transcrit $\mathrm{M} \in(\mathrm{NI})$ ), il s'agit d'un $\mu$ ligaturé à un o en hauteur (pour $\mu o ́(v \alpha))$.

\section{O.Edfou Copte 37 [AD]}

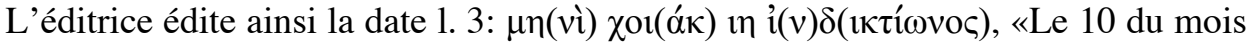
de Choiak de la $8^{\mathrm{e}}$ année de l'indiction». Dans les autres documents, le chiffre de l'année est indiqué après la mention de l'indiction. C'est ici aussi de cette manière qu'il faut comprendre le texte: in est donc le chiffre du jour et le chiffre de l'année doit se lire ensuite. On peut éditer $\mu(\eta v i) \operatorname{Xot}(\alpha \kappa)$ in $\mathfrak{i}(v) \delta(1 \kappa \tau i ́(\omega v o \varsigma) \varepsilon$ ou $\varsigma$, «Au mois de Khoiak, le $18,5^{\mathrm{e}}$ ou $6^{\mathrm{e}}$ année de l'indiction.»

\section{O.Edfou Copte 38}

L. 2: NGaYON est impossible. Le mot se finit par Tı. [JLF]

L. 3: $\pi(\alpha \hat{v}) v(1) \rightarrow \Pi(\alpha) v(v \imath)$. [AD]

\section{O.Edfou Copte 39 [JLF]}

L. 4 et 5: тpanezd $\rightarrow$ тpaneızd. Pour cette orthographe, cf. Förster, WB, s.v. $\tau \rho \alpha ́ \pi \varepsilon \zeta \alpha$. 


\section{O.Edfou Copte 40 et 42 [JLF]}

Le texte de l'éd. d'O.Edfou Copte 40, 1-4 est le suivant:

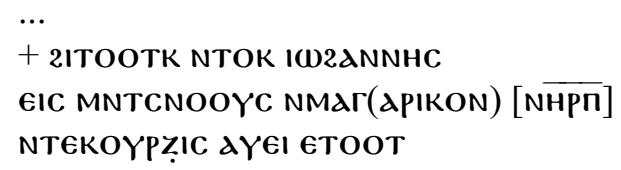

«Par tes soins à toi, Jôhannês, voici que douze mag(arika) de vin de Tekourzis me sont parvenus.»

On lit, à la première ligne (juste au-dessus de оотK), kọ.pczıc devant lequel rien n'est lisible sur la photo. On a la répétition d'une séquence qui se retrouve à la 1. 4: Nтекоүрzıı qu'il faut lire sans aucun doute NTEкоүрсzıc. L'éditrice propose d'y voir le toponyme Tekoursis qui serait une détermination du vin. Même si, d'après la photo, rien ne semble avoir été écrit après Mar(apIKON), les parallèles incitent à sous-entendre NHPn. On comparera O.Edfou Copte 42, où je propose de retrouver le même nom. En voici les deux premières lignes d'après l'édition:

\section{[+ EIC COOY] NMATAPIKON NHPח NTOK AP()IC)}

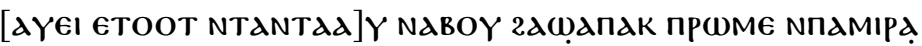

«[Voici que six] magarika de vin, toi, Archich [nous sont parvenus. Nous les avons remis] à Abu Haôapak, l'homme de l'émir.»

L'éditrice s'étonne à juste titre de la place de la séquence NTOK $\lambda P() ı)$; elle voit dans $\lambda \mathbf{p}(\omega)(\omega)$ un nom propre signifiant «pois chiche». Or «pois chiche» se dit

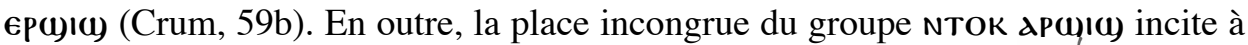

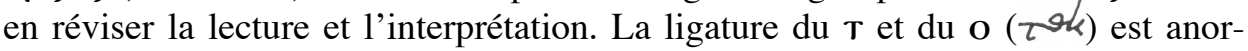
male; on trouve en revanche communément le même tracé pour $\epsilon$, notamment après T (pour se limiter au dossier, cf. O.Edfou Copte 45, 3; 57, 4), mais aussi

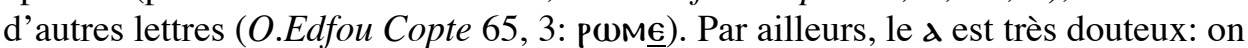
distinguerait plus volontiers deux lettres, dont la première ressemble aux autres $\mathbf{O}$, très ouverts, du texte et la seconde à un $\gamma$ (encore qu'on attendrait ensuite une haste descendante). Je propose donc de lire NTEKoY.P()ı) et de voir dans ce mot une variante du NTEKoYPCZıc d'O.Edfou Copte 40: on sait que $\omega$ est communément transcrit en grec par le groupe $\sigma \zeta$ en début ou en milieu de mot et par $\varsigma$ en fin de mot. Le rédacteur a donc adopté, dans O.Edfou Copte 40, une orthographe grécisante.

Je serais d'avis de délimiter le toponyme au seul koүpczıc/koү.p()ı) précédé de la conjonction NTE introduisant le génitif (comme dans le parallèle édité par St. Jakobielski cité et revu par A. Delattre ci-dessus, p. 84: eIC WOMNTe NBdIC)OMNT $\overline{\text { NHPT }}$ | NTE חME2CNAY NEIOOM NTd2(OP). Cela expliquerait que, dans l'ajout au-dessus d'O.Edfou Copte 40, 1, on ne lise que koypczic: le texte 
du reçu a été surmonté par le rédacteur ou celui qui l'a archivé du mot qu'il considérait comme le plus important, en l'occurence la provenance du vin, de façon à le mettre en exergue. Kouršiš est donc un microtoponyme nouveau de l'Apollonopolite, qu'il faut sans doute lire également dans O.Edfou Copte 45, 3 (voir ci-dessous).

Puisque NTE koY.P()ı) est une détermination du vin, il faut restituer ailleurs le nom du destinataire du reçu, qui, conformément au formulaire des reçus d'Edfou, devrait se trouver en début de document. Il se trouve que l'éditrice restitue, à la première ligne, une lacune anormalement courte par rapport à celles des deux lignes suivantes.

On obtient ainsi le texte suivant:

\section{O.Edfou Copte 40, 1-5}

\section{KOY.PCZIC}

+ 2ITOOTK NTOK IW2ANNHC

EIC MNTCNOOYC NMAT(APIKON) $\langle\overline{N H P \Pi}\rangle$

NTEKOYPCZIC AYEI ETOOT

5 NTAKTAdY NMAPI2AM T6AYO[N]

«Kouršiš. Par tes soins, Jôhannês, voici que douze magarika <de vin> de Kouršiš me sont parvenus, que tu as donnés à Mariham, la servante.»

\section{O.Edfou Copte 42}

[+ 2ITOOTK NTOK NN EIC COOY] NMAгаPIKON NHPח NTE KOY.P()IC)

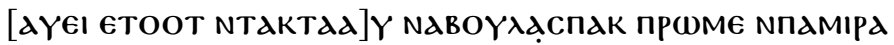

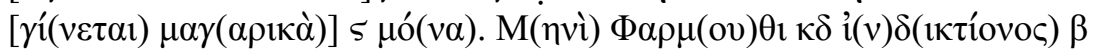

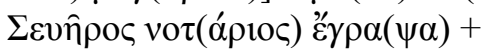

"Par tes soins, NN, voici que six magarika de vin de Kouršiš me sont parvenus, que tu as donnés à Aboulaspak, le serviteur de l'émir. Total: 6 magarika seulement. Écrit par moi, Sevêros, notarios, le 24 du mois de Pharmouthi de la $2^{\mathrm{e}}$ année de l'indiction.»

2 NTAKTAd]Y: préférable à NTANTAd]Y (éd.: «nous les avons remis à ...»), qui apporterait une information non nécessaire dans un reçu, et qui est démenti par O.Edfou Copte 40, 5 et 45, 4 (tel qu'il est restitué ci-dessous). Sevêros veut signifier par là qu'il enregistre le versement des 6 magarika de vin au compte du destinataire du reçu, même si celui-ci les a remis physiquement à quelqu'un d'autre (probablement à la demande de Sevêros). Il faut également corriger NTd[NTAdC en NTd[KTdaC en O.Edfou Copte 47, 6.

двоүласспдк: le $\lambda$ est réalisé de façon très rapide comme dans $\underline{\lambda}$ or(oc) en O.Edfou Copte 60, 1. Ce nom pourrait être la transcription du nom arabe, 
fréquemment attesté au début de l'Islam, Abû l-Asbag ${ }^{17}$ avec passage de $b$ à $\mathrm{n}$ et, puisque le ghayn peut être rendu par un $\Gamma$ (cf. P.Lond. IV 1441, 82: M $\omega \alpha \gamma \varepsilon \rho<$ ar. Muǵayyir ${ }^{18}$ ), passage de $\Gamma$ à $\mathrm{K}$, autrement dit un assourdissement des deux consonnes. Cette personne est probablement un des esclaves de l'émir (пршмє NחגMipa).

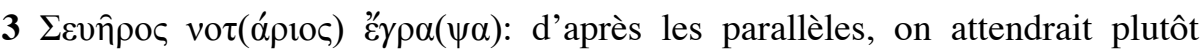

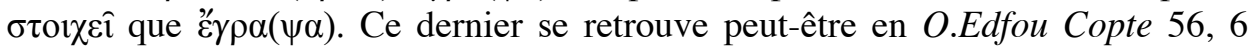

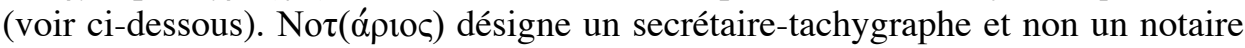
(cf., ci-dessus, note à O.Edfou Copte 36-65).

\section{O.Edfou Copte 41 [AD-JLF]}

Le parallèle O.Vars. Inv. 139901 permet d'interpréter la 1. 4: NTEıє introduit les indications relatives à la distribution du vin. Deux bénéficiaires sont nommés: прєєітдк, sans doute un micro-toponyme, et les NPM пкג2, «les hommes de la terre». On notera qu'outre les quatre magarika et les deux tripla, une autre quantité de vin, introduite par le MN de la 1.3, était indiquée dans la lacune de la 1. 4. Au début des lignes 6 et 7, les lacunes devaient être importantes et d'autres bénéficiaires devaient être mentionnés. On peut dès lors rééditer le document ainsi.

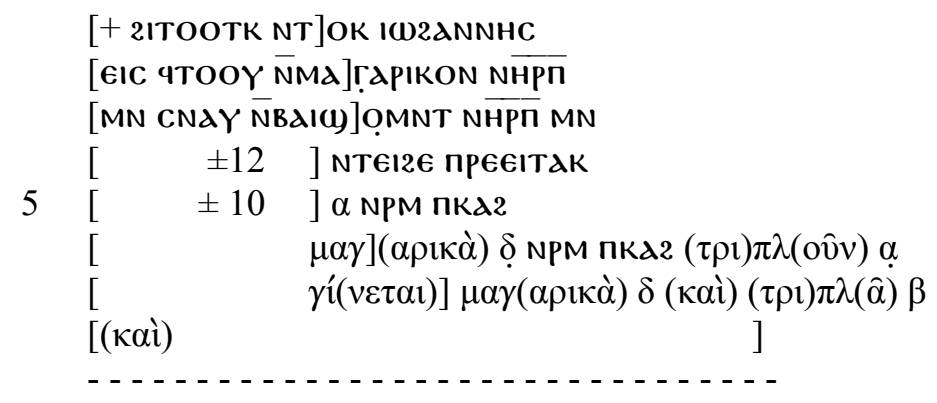

«Par tes soins à toi, Jôhannês, voici que quatre magarika de vin et deux baichomnt de vin et [...] de cette manière: (pour) Preeitak [...] 1; (pour) les hommes de la terre [...] 4 magarika; (pour) les hommes de la terre 1 triploun [...] Total: 4 magarika, 2 tripla et [...].»

\section{O.Edfou Copte 44}

L. 2: [BIKT]

L. 3: il faut mentionner la présence de traces d'une troisième ligne. [AD]

\footnotetext{
17 Je remercie Jean-Michel Mouton et Yussef Ragheb de m'avoir suggéré ce rapprochement.

18 Je remercie Jean Gascou de cette référence.
} 


\section{O.Edfou Copte 45 [AD]}

L. 2: Ma. [ $\rightarrow$ Marạ[PIKON]. Le document est donc à interpréter comme un reçu de magarika de vin. 42 .

L. 3: NTEkoY! [ $\rightarrow$ NTEkoYp[('ı)], cf., ci-dessus, note à O.Edfou Copte 40 et

L. 4: $\mathrm{NTdK}[\rightarrow \mathrm{NTdK}[\operatorname{Tad\gamma }+$ nom du bénéficiaire], cf., ci-dessus, note à O.Edfou Copte 42.

\section{O.Edfou Copte 47 [JLF]}

L. 4: c[IXBANOC $\rightarrow$ c! $]$ [BaNOC.

L. 6: cf., ci-dessus, note à $O . E d f o u$ Copte 42, 2.

\section{O.Edfou Copte 48 et 49 [AD-JLF]}

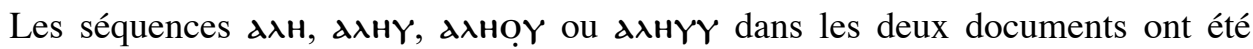
comprises comme une forme de $\lambda \lambda \epsilon\}$, qui signifie «blanc» en copte. Les deux ostraca porteraient donc des comptes de vin blanc. En réalité, les textes sont purement grecs et il faut lire la séquence $\alpha \lambda H$ et ses variantes $\alpha \lambda \eta(v o v)$ ou $\alpha$ $\lambda \eta v 0 v \hat{~} 1^{\text {ère }}$ cuvée» ${ }^{19}$. Voir, comme textes parallèles, SPP X 255 (Arsinoïte, sans date [époque arabe d'après les abréviations]), P.Iand. IV 61 (prov. inconnue, IV ${ }^{\mathrm{e}}$ s.). On pourrait avoir un autre exemple dans le présent dossier: voir, ci-dessous, note à $O$.Edfou Copte 105.

La mention de différentes «cuvées» se lit aussi dans des documents coptes, comme P.Pisentius 33, 3, où il est question de vin blanc «de première cuvée»

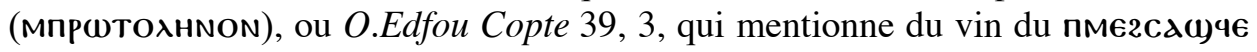
NGıOM, «de la septième cuvée».

\section{O.Edfou Copte 48}

\begin{tabular}{|c|c|c|c|}
\hline 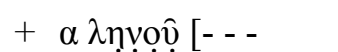 & $(\tau \rho \imath) \pi \lambda(\hat{\alpha})$ & $\ll 1^{\text {ère }}$ cuvée de $\ldots$ & ... tripla \\
\hline$\beta$ & $(\tau \rho \mathrm{l}) \pi \lambda(\hat{\alpha})$ & $2^{\mathrm{e}}$ cuvée & ... tripla \\
\hline$\alpha \lambda \eta$ ỵôิ traces & {$[(\tau \rho \imath)] \pi \lambda(\hat{\alpha})[$} & $1^{\text {ère }}$ cuvée de ... & ... tripla \\
\hline$\beta$ & $(\tau \rho) \pi \lambda(\hat{\alpha})[$ & $2^{\mathrm{e}}$ cuvée & ... tripla \\
\hline$\alpha \lambda \eta v o \hat{v}$ & $(\tau \rho i) \pi \lambda(\hat{\alpha})$ & $1^{\text {ère }}$ cuvée de ... & ... tripla \\
\hline$\beta$ & $(\tau \rho \imath) \pi \lambda(\hat{\alpha})$ & $2^{\mathrm{e}}$ cuvée & ... tripla \\
\hline$\gamma$ & $(\tau \rho !) \pi \lambda(\hat{\alpha})$ & $3^{\mathrm{e}}$ cuvée & ... tripla \\
\hline 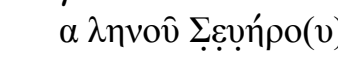 & )$^{?}(\tau \rho \mathrm{t}) \pi \lambda(\hat{\alpha})$ & $1^{\text {ère }}$ cuvée de Sevêros? & ... tripla \\
\hline$\beta$ & $(\tau \rho \imath) \pi \lambda(\hat{\alpha})[$ & $2^{\mathrm{e}}$ cuvée & ... tripla \\
\hline $\begin{array}{c}10 \alpha \lambda \eta v o v \ldots . . .() \\
\text { traces }\end{array}$ & {$[(\tau \rho \mathrm{l}) \pi \lambda(\hat{\alpha})$} & $1^{\text {ère }}$ cuvée de $\ldots$ & ... tripla...» \\
\hline
\end{tabular}

19 Sur l'interprétation du mot $\lambda$ nvóc, cf. J.-P. Brun, Archéologie du vin et de l'huile dans l'Empire romain, Paris, 2004, p. 146-147. 


\section{O.Edfou Copte 49}

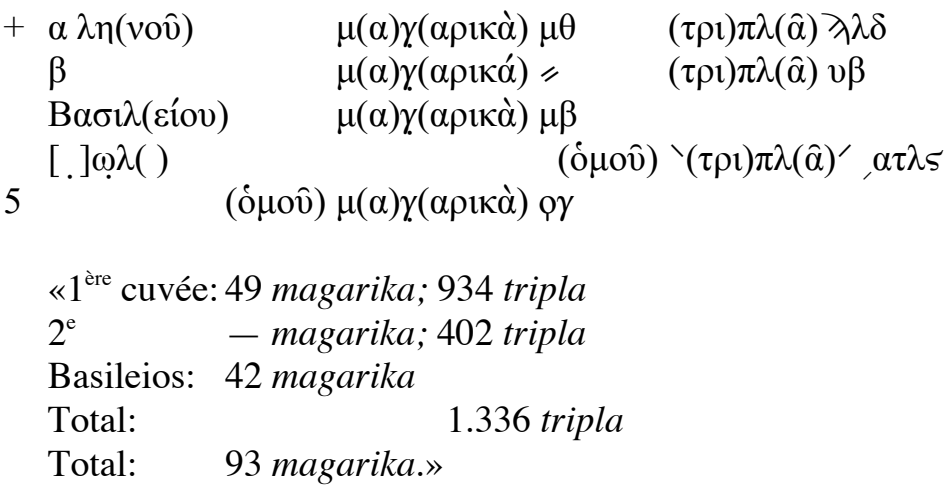

1-3, $5 \mu(\alpha) \gamma\left(\alpha \rho\right.$ có $^{2}$ : on lirait plutôt $\mu() \tau($ ) ou $\mu$. $\tau($ ). L'éd. a d'ailleurs lu $\mu(\hat{\varepsilon}) \tau(\rho \alpha)$ à la 1 . 3 et $\mu \alpha \gamma(\alpha \rho$ ı́ $)$ aux autres. Il faut bien pourtant que ce soit le même mot qui apparaisse aux 1. 1-3 et 5 puisque cette dernière donne le total des trois premières. Par ailleurs, on hésite à lire partout $\mu(\hat{\varepsilon}) \tau(\rho \alpha)$ dans la mesure où le metron n'est pas attesté comme une mesure du vin à Edfou. Le seul contreexemple, P.Apoll. 93, 1, 3, $\mu(\varepsilon ́ \tau \rho \alpha)$, doit être corrigé puisqu'on lit clairement sur le papyrus $\gamma$, autrement dit $\mu(\alpha) \gamma\left(\alpha \rho \alpha_{\alpha}\right)$. C'est cette dernière mesure qui se rencontre dans le présent dossier. L'autre solution serait de lire $\mu(\varepsilon v) \tau$ (cf. p.ex. P.Heid. II 214, 40); mais cette mesure n'est pas attestée pour l'instant à Edfou.

2 : : équivaut à un 0.

3 B $\alpha \sigma i \lambda$ (cíov): nom d'un autre viticulteur. Ce nom revient à plusieurs reprises dans le dossier.

$\mu \beta$ : le $\beta$ est une correction sur un $\delta$; on s'attendrait à l'inverse d'après le total $(49+44=93)$.

4 [.] $\omega \lambda($ ( ): il est possible que le scripteur ait voulu écrire $(\tau \rho \mathrm{\imath}) \pi \lambda(\hat{\alpha})$ et qu'il n'ait pas terminé le $\pi$ (qui du coup, sans son trait horizontal, ressemble à un $\omega$ ), se rendant compte qu'il n'avait pas respecté l'alignement avec les autres $(\tau \rho \imath) \pi \lambda(\hat{\alpha})$.

\section{O.Edfou Copte 50}

L. 2: $\lambda \Pi \lambda \rightarrow \dot{\alpha} \beta \beta(\hat{\alpha})$. [AD]

L. 4: o(mọ̣c) $\rightarrow \Lambda \varepsilon^{\prime}(\alpha)$ ? Cf. la proposition de lecture dans 75, 1. [AD]

L. 7: $x \in \rightarrow \Theta \varepsilon o ́ \delta(\omega \rho \circ \varsigma)$. [AD-JLF]

\section{O.Edfou Copte 51 [AD]}

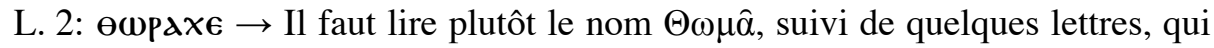
représentent peut-être un patronyme ou un anthroponyme. 


\section{O.Edfou Copte 52 [JLF]}

On peut dater paléographiquement le document $\mathrm{du} \mathrm{VI}^{\mathrm{e}}$ siècle.

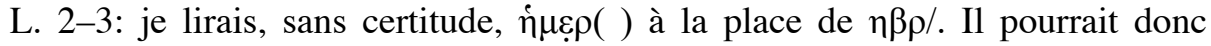
s'agir de rétributions en vin de jours de travail.

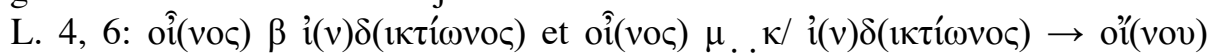

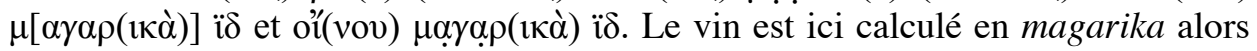

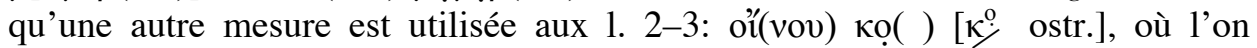

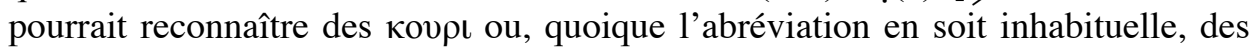
кó $\lambda \lambda \alpha \theta \alpha$.

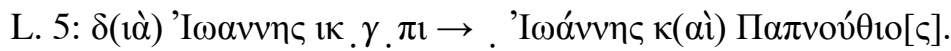

\section{O.Edfou Copte 53}

L. 1: $\Pi(\alpha \hat{v}) v(\imath) \varsigma \lambda(\alpha) \chi(\alpha ́ v \omega v) \rightarrow \Pi(\alpha) v(v \imath) \varsigma \lambda(\alpha) \chi(\alpha v o \sigma \pi \varepsilon ́ p \mu o v)$. Pour le sens de $\lambda \alpha \chi \alpha v o ́ \sigma \pi \varepsilon \rho \mu o v$, cf. en dernier lieu F. Morelli, «Il $\lambda \alpha \chi \alpha v o ́ \sigma \pi \varepsilon \rho \mu o v$, il

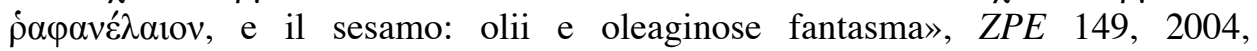
p. 138-142. [JLF]

—: $\beta \gamma \rightarrow \beta$. Même erreur en O.Edfou Copte 89, 4; 97, 3; 8. [JLF]

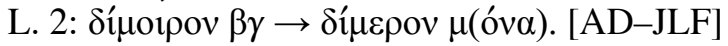

\section{O.Edfou Copte 54 [JLF]}

L. 2: $\alpha \gamma \mu \kappa \rho \imath \theta(\hat{\eta} \varsigma) \dot{\alpha} \rho \tau(\alpha \dot{\alpha} \beta \alpha \imath) \rightarrow{ }^{`} \mathrm{~A} \theta v(\rho) \lambda \kappa \rho \imath \theta(\hat{\eta} \varsigma) \dot{\alpha} \rho(\tau \alpha \dot{\beta} \beta \alpha \imath)$.

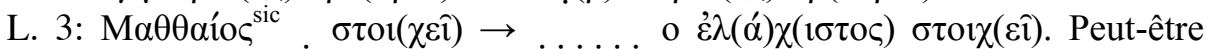

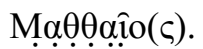

\section{O.Edfou Copte 55 [JLF]}

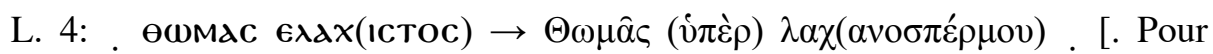

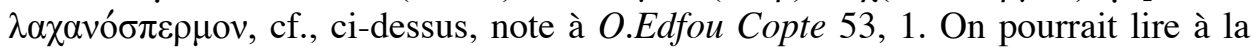
fin $(\ddot{\eta} \mu 1 \sigma v)$ sc. $\dot{\alpha} \rho \tau(\dot{\alpha} \beta \eta \varsigma)$ ?

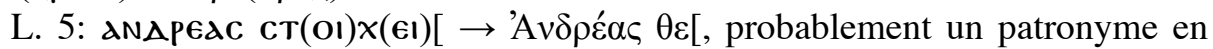
$\Theta \varepsilon 0_{-}$.

\section{O.Edfou Copte 56}

L. 2: пุגTEI $\rightarrow 2 \lambda$ TEI. [AD]

L. 5: $\operatorname{m\epsilon }(\mathrm{NI}) \phi \lambda \omega(\phi ı) \rightarrow \mu o ́(v \alpha) \Phi(\alpha) \omega(\varphi \imath)$; cf., ci-dessus, note à $O . E d f o u$ Copte 36-43, 55. [AD]

L. 6: $\operatorname{\epsilon rp}(\lambda \phi н) \rightarrow \stackrel{\varepsilon}{\varepsilon} \gamma \rho \alpha(\psi \alpha)$, cf. O.Edfou Copte 42, 3 (voir ci-dessus). [ADJLF]

\section{O.Edfou Copte 60}

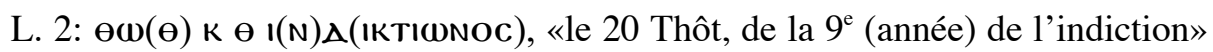
$\rightarrow \Theta \omega(\theta) \kappa \theta \mathfrak{i}(v) \delta(1 \kappa \tau i ́ \omega v o \varsigma) \gamma$, «Le 29 Thôt de la $3^{\mathrm{e}}$ année de l'indiction». [ADJLF] 
Entre 1. 2 et 3: lire probablement: ọ(vँ $\tau \omega \varsigma)-[\mathrm{JLF}]$

L. 5: 2wOYT $\rightarrow$ 2OOYT. [JLF]

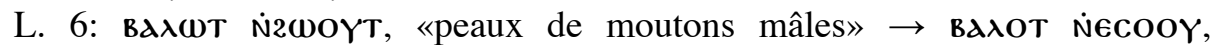
«vêtements en peau de mouton». Dans ce texte, comme dans P.Bal.332, les

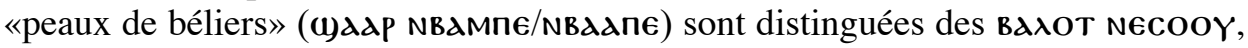
cf. Crum, Dict., p. 38a. [AD]

\section{O.Edfou Copte 61 [AD-JLF]}

Ce document, ainsi qu'O.Edfou Copte 96 et 100-102, comporte l'abréviation $\breve{\chi}$, que l'éditrice résout systématiquement $\chi(\rho v \sigma o v) v(o ́ \mu 1 \sigma \mu \alpha)$. Cette interprétation se heurte à plusieurs obstacles: la lettre sur le $\chi$ ne ressemble pas à un $v$, mais plutôt à un $v$; on ne voit pas ce que viendraient faire des solidi sur le même plan que des artabes d'orge dans le présent compte; enfin, en O.Edfou Copte 96, 5, la somme de solidi (358) serait anormalement haute. Partout ailleurs, $\chi(\rho v \sigma o v) v($ ó $\mu 1 \sigma \mu \alpha)$ est écrit $\chi \bar{\rho}, \chi \rho^{\circ}$ ou $\chi \rho$ suivi de ${ }^{\circ}$ ou $\bullet$ (cf. O.Edfou Copte 88,$3 ; 89,3 ; 90,3 ; 91,6$; 95 , 2). La même abréviation apparaît dans O.Vind. Copt. 136, 2, 4 et 5 (= O.Crum ST 441), ainsi que dans SB Kopt. III $1444^{20}$. Les propositions de résolution dans cette

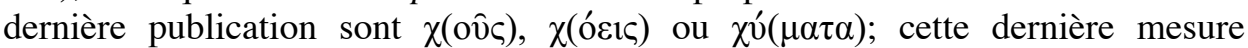
semble la plus plausible ${ }^{21}$. On remarquera que, d'après O.Edfou Copte 61, il semble y avoir un rapport de 1 à 10 entre les artabes d'orge et les chumata si on lit ainsi les 1. 3-4:

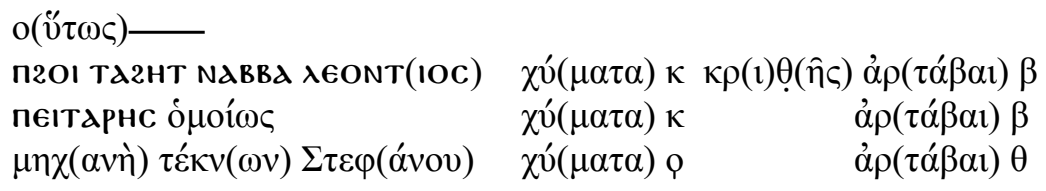

\section{O.Edfou Copte 62 [JLF]}

Ce document concerne, au moins partiellement, les mêmes terrains que le

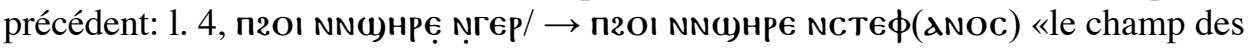
enfants de Stephanos» (cf. O.Edfou Copte 61, 4).

L. 3: т. Ха̣ [ $\rightarrow$ тגхגM, anthroponyme, que l'on rapproche habituellement de TA2גM.

\section{O.Edfou Copte 67}

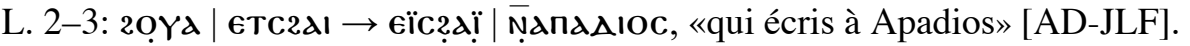

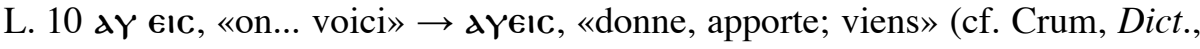
p. 19b) [AD].

20 A. Di Bitonto Kasser, «Ostraca copti a Deir el Gizaz», Aegyptus 74, 1994, p. 75-122, en part. p. 81 .

${ }^{21}$ Sur la mesure, cf. N. Kruit, «Local Customs in the Formulas of Sales of Wine for Future Delivery (A Supplement to P.Heid. V)», ZPE 94, 1992, p. 167-184, en part. p. 172. 


\section{O.Edfou Copte 68 [AD-JLF]}

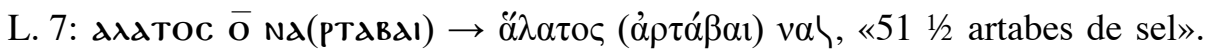

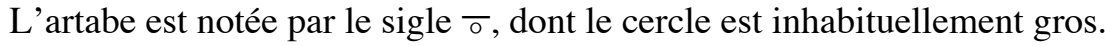

\section{O.Edfou Copte 70 [AD-JLF]}

L. 1: пגatonioc. On peut se demander s'il ne faut pas lire le nom nגatonic, qui est un nom de femme (O.Douch. II 58, 2; P.Oxy. XIV 1647, 1-2 et 1721, 1).

\section{O.Edfou Copte 75 [JLF]}

Il ne s'agit pas d'un reçu en copte, mais d'un ordre de versement, probablement de blé, en grec. Je propose (avec prudence) de reconstituer ainsi le texte:

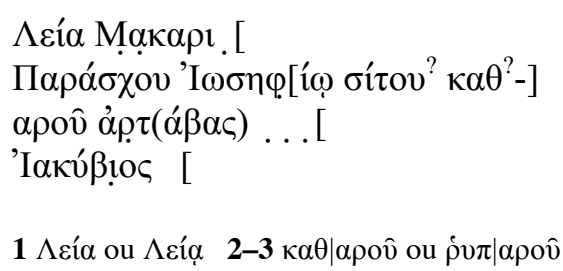

\section{O.Edfou Copte 79 [AD-JLF]}

L. 1-2: $\overline{\text { NXP!CT! }}$ |dNoc. On ne trouve pas d'attestation de cet anthroponyme dans la documentation. La lecture incertaine du début du mot inciterait à ne pas accepter ce nouveau nom.

\section{O.Edfou Copte 80}

L. 2: cıхвє̣ctpoc. Le nom, dont la fin est très difficile à déchiffrer, n'apparaitt pas à notre connaissance à Edfou, ni ailleurs dans la documentation papyrologique. Une lecture ci৯BaNOC semble dès lors préférable. [JLF]

L. 3: THP дKP . єI[ $\rightarrow 2 \mathrm{HP} \lambda K \lambda \in I \Delta \mathrm{H}$ traces. [AD]

\section{O.Edfou Copte 88}

L. 1: ce qui a été lu comme une croix est sans doute plutôt le $\theta$ de $\Sigma \varepsilon v o v ́ \theta(10 \varsigma)$, écrit en suspension au-dessus de la ligne. En conséquence, il n'y a pas de 1. 1. [AD]

La 1.2 (= 1. 1) a été lue par l'éditrice $\Sigma \varepsilon v o v \theta(i ́ o v)$ 'İ̀ Пó $\rho$, mais des traits d'abréviations sont visibles au-dessus de l' $\omega$ et du $\rho$; par ailleurs, le nom Por est

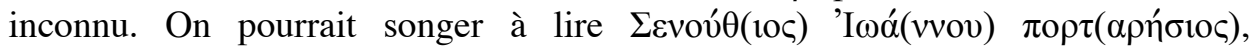
«Sénouthios, fils de Jôhannês, le portarensis». [AD]

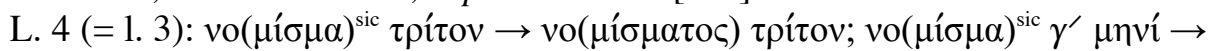

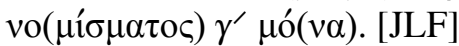

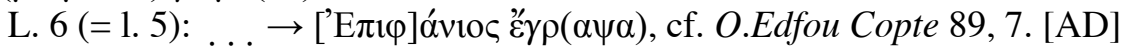




\section{O.Edfou Copte 89 [AD-JLF]}

L. 3: $(v o \mu i ́ \sigma \mu \alpha)^{\text {sic }} \rightarrow$ vo( $\left.\mu i ́ \sigma \mu \alpha \tau o \varsigma\right)$. [JLF]

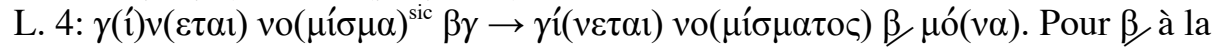
place de $\beta \gamma$, cf., ci-dessus, note à O.Edfou Copte 53,1 . [JLF]

L. 5: '̇ं $\mathbf{i}^{\prime} \varphi(\mathrm{l}) \rightarrow$ 'E $\pi \iota \varphi$ (ce qui a été pris pour une barre d'abréviation est l'apex du $\varphi)$. [JLF]

L. 7: $\sigma \tau \mathrm{o}^{\prime} \chi(\varepsilon \varepsilon)^{\mathrm{sic}}+\rightarrow \stackrel{\prime}{\varepsilon} \gamma \rho \alpha(\psi \alpha)+.[\mathrm{AD}]$

\section{O.Edfou Copte 90}

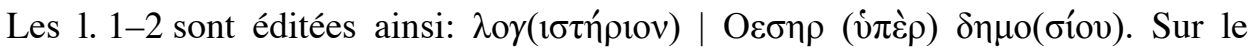
modèle des autres reçus, il est préférable de lire un anthroponyme au début du

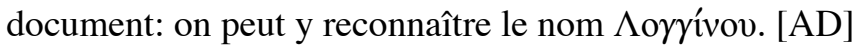

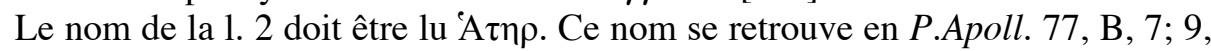

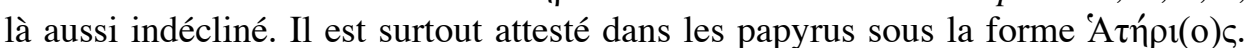
L'aspiration est assurée par le copte 2ג THP (O.Medin. Habu Copt. 28,5). Voir, cidessous, note à $O . E d f o u$ Copte 101, 7. [JLF]

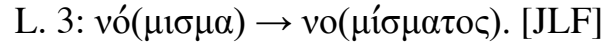

L. 4: $\Theta \dot{\omega} \theta \rightarrow \Theta \omega(\theta)$. [JLF]

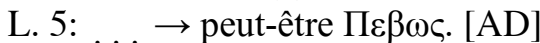

\section{O.Edfou Copte 91}

Le texte doit faire parallèlement l'objet de rééditions (par Jean Gascou dans les Mélanges Cécile Morisson et par Klaas A. Worp dans le BASP).

\section{O.Edfou Copte 92 [AD-JLF]}

Rien dans ce texte n'impose d'y voir un document copte. Il pourrait s'agir d'un texte paralittéraire grec.

\section{O.Edfou Copte 93}

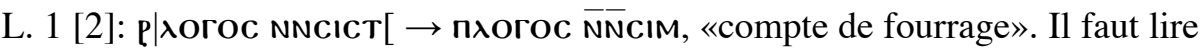
ici le titre du document, qui a visiblement été ajouté dans un second temps, en petits caractères. On notera aussi qu'il n'y a pas de trace de lettres au-dessus de cette ligne. Le texte commençait donc initialement 1 . 2 , où il faut donc lire une croix plutôt qu'un $†$. À côté des chiffres $\Delta$ et $\mathbf{B}$ des 1.2 et 3 , on distingue des lettres de petit format, sans doute la suite du titre de la 1. 1. [AD-JLF]

L. 3 [4]: ceinOYeIOC $\rightarrow$ cenOYeIOC (ce qui a été pris pour un iota est le bas de la croix de la l. précédente). [JLF]

L. 4 [5]: $2 \lambda \lambda \epsilon() \mathrm{H} \mid, Y \rightarrow 2 \lambda \lambda \epsilon(\omega) \mathrm{HY}$. [JLF]

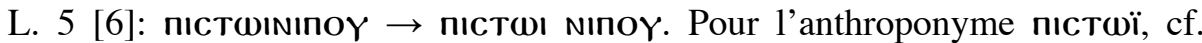
O.Douch I 49, 1. [JLF]

L. 7 [8]: la ligne se termine par le chiffre H. [AD-JLF] 


\section{O.Edfou Copte 94 [JLF]}

Ostracon grec d'époque romaine ( $\mathrm{III}^{\mathrm{e}} \mathrm{s}$.). On peut lire ainsi les 1. 5-7:

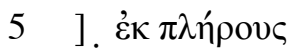

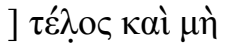

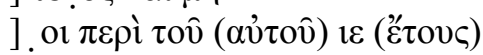

$7 \zeta \varepsilon \zeta$ ostr.

\section{O.Edfou Copte 95 [AD-JLF]}

On peut relire ainsi le document 95:

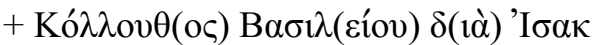

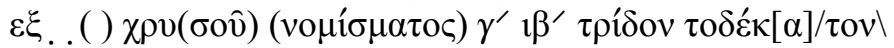

. . i $(v) \delta(1 \kappa \tau i ́ \omega v o \varsigma) \beta A \pi \alpha \imath$. $\sigma \tau \circ \chi(\varepsilon \hat{\imath})+$

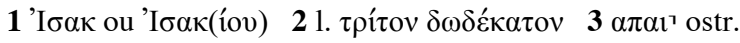

\section{O.Edfou Copte 96 [JLF]}

$\dot{\mathrm{o} \mu \mathrm{o}(\hat{i} \omega \varsigma) \operatorname{Ko\sigma \mu }[(\hat{\alpha} \varsigma)] \chi \chi{ }^{\prime}(\mu \alpha \tau \alpha) \rho \alpha}$

$[\ldots . . .$.

(кai) vs

[(ópô) $\chi \underline{v}(\mu \alpha \tau \alpha) \ldots]$

5 (o $\mu \mathrm{ov}) \chi \hat{v}(\mu \alpha \tau \alpha) \tau v \eta$

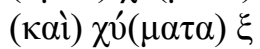

3, 6 > 4-5 $\varnothing$ ostr. $5 \check{\chi}$ ou $\chi$ ostr. $6 \xi$ ou $\zeta$

Ce qui précédait la ligne 1 totalisait $200 \chi \chi(\mu \alpha \tau \alpha)$; sur l'abréviation, cf., cidessus, note à O.Edfou Copte 61.

\section{O.Edfou Copte 97 [JLF]}

Rien n'impose de voir dans ce document les comptes de la pagarchie, qui n'étaient certainement pas consignés sur ostraca.

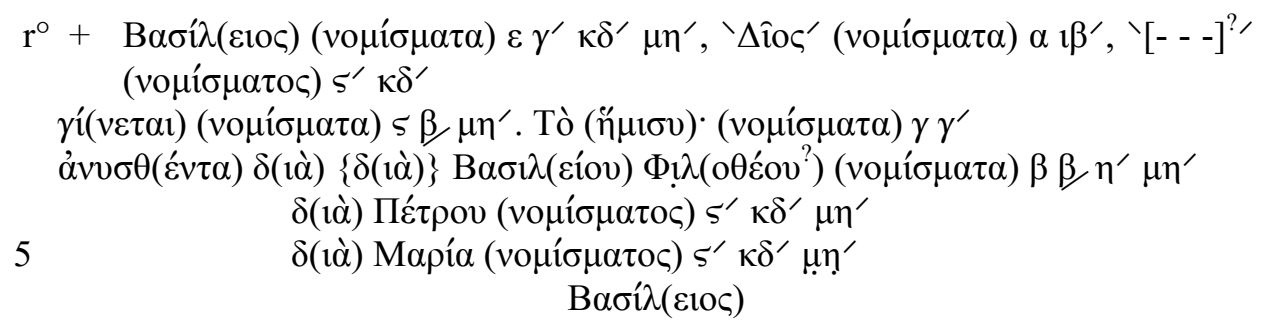


$\mathrm{v}^{\circ}\left[\begin{array}{ll}\quad \pm 9 & ] \varsigma^{\prime} \kappa \delta^{\prime}\end{array}\right.$

traces? $\quad \gamma^{\prime}(v \varepsilon \tau \alpha)(v o \mu i ́ \sigma \mu \alpha \tau \alpha) \varsigma \gamma^{\prime}{ }_{1} \beta^{\prime} \mu \eta^{\prime}$

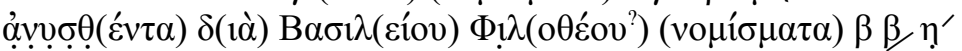

10 traces de 2 ou 3 lignes (effacées?)

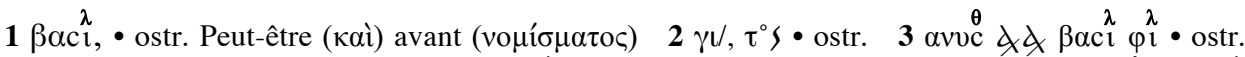

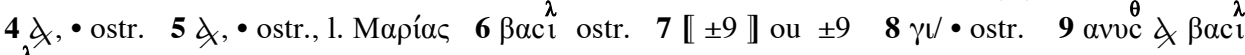
$\varphi \mathrm{i} \cdot$ ostr.

«Basileios: 5 1/3 1/24 1/48 nomismata; Dios: 1 1/12 nom.; [...:?] 1/6 1/24 nom. Total: 6 2/3 1/48 nom. La moitié: 3 1/3 nom.

Perçu par Basileios fils de Phil(otheos'): 2 2/3 1/8 1/48 nom.

- par Petros: 1/6 1/24 1/48 nom.

- par Maria: 1/6 1/24 1/48 nom.

$\left(\mathrm{v}^{\circ}\right)$

$\left[\ldots{ }^{2}\right] \quad 1 / 61 / 24$

Total: 6 1/3 1/12 1/48 nom.

Perçu par Basileios fils de Phil(otheos?): 2 2/3 1/8 nom.

$[\ldots ?] »$

\section{O.Edfou Copte 98 [JLF]}

L. 3: ] $\quad \alpha \mu \varepsilon \delta \rightarrow M \alpha \alpha \mu \varepsilon \delta$.

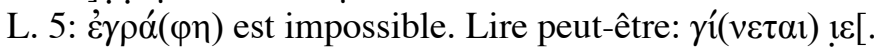

\section{O.Edfou Copte 99}

L. 2-6: vó $(\mu 1 \sigma \mu \alpha) \rightarrow$ vo( $\mu$ í $\sigma \mu \alpha \tau o \varsigma)$. [JLF]

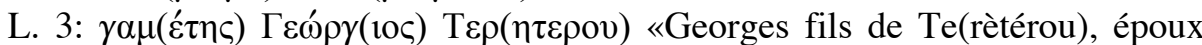
(?)» $\rightarrow \gamma \alpha \mu(\varepsilon \dot{\tau} \tau) \Gamma \varepsilon \omega \rho \gamma\left(\right.$ íov) $\Pi \varepsilon \varepsilon_{\tau}(\mathrm{ov})$ «l'épouse de Georgios fils de Petros». Sur la photo, on lirait plutôt $\tau \varepsilon \tau \rho($ ) que $\pi \varepsilon \tau \rho($ ), mais je ne connais pas de nom commençant ainsi (le nom TeTPdC enregistré par M. Hasitzka, Namen in koptischen dokumentarischen Texten, avec un renvoi à O.Medin. Habu Copt., est une coquille pour TEPגC). [JLF]

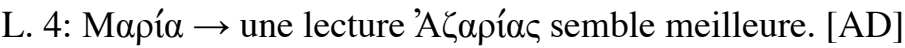

L. 6: $\Lambda$ o $\gamma \gamma \mathrm{ivo}(\varsigma)^{\text {sic }} \rightarrow \Lambda$ o $\gamma \gamma$ ívo(v). [JLF]

\section{O.Edfou Copte 100}

Au sujet de l'abréviation $\breve{\chi}$ pour $\chi v ́(\mu \alpha \tau \alpha)$, cf., ci-dessus, note à O.Edfou Copte 61.

\section{O.Edfou Copte 101}

L. 2-7: au sujet de l'abréviation $\breve{\chi}$ pour $\chi v((\mu \alpha \tau \alpha)$, cf., ci-dessus, note à $O . E d f o u$ Copte 61.

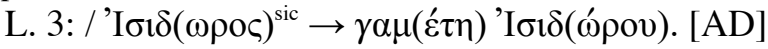

L. 4: ' $\mathrm{I} \alpha \kappa(\kappa \omega \beta \mathrm{\beta} \varsigma)^{\mathrm{sic}} \rightarrow \operatorname{M\alpha \kappa }(\alpha \rho \varepsilon)$ ? [JLF] 


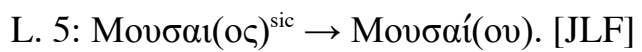

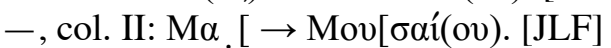

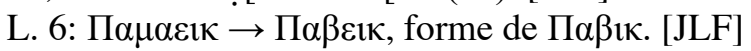

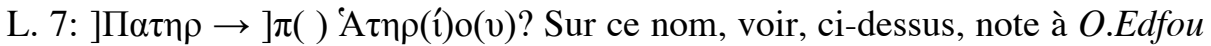
Copte 90, 2. Ici, le omicron qui surmonte le $\rho$ sous la forme d'un point (ce que n'a pas vu l'éd. d'après sa note aux 1. 6-7) implique que le nom est décliné contrairement à O.Edfou Copte 90, 2 et P.Apoll. 77, B, 7; 9. [JLF]

\section{O.Edfou Copte 102}

Au sujet de l'abréviation $\breve{\chi}$ pour $\chi v ́(\mu \alpha \tau \alpha)$, cf., ci-dessus, note à O.Edfou Copte 61.

\section{O.Edfou Copte 105 [JLF]}

Il pourrait s'agir d'un compte de cuvées. Sous-entendre alors $\lambda \eta v o v$ après $\alpha, \beta, \gamma$ et peut-être $[\delta]$.

\section{O.Edfou Copte 107 [AD]}

On remarquera que les lacunes sont probablement plus importantes que ne l'indique l'édition, où la lacune à droite est tantôt inexistante, tantôt d'une, deux ou quatre lettres.

Je proposerais plutôt de voir dans $х$ גїт2н (1.7) une forme de $6 \omega$ T2 ( $\chi \omega T 2$, cf. 6at2€, «hole», Crum, Dict., p. 834b) et de comprendre «sans trou, sans fêlure».

\section{O.Edfou Copte 120 [JLF]}

L. 3: k.т. $\rightarrow$ ḳıTe. Pour ce nom, cf. O.Edfou Copte 123, 6 et, ci-dessous, note à 125,2 .

\section{O.Edfou Copte 125 [JLF]}

L. 2: . . . [ $\rightarrow$ kïтє. Pour ce nom, cf. O.Edfou Copte 123, 6 et, ci-dessus, note à 120,3 .

\section{O.Edfou Copte 126 [JLF]}

Il ne s'agit pas d'un «bon», mais d'un dipinto amphorique donnant le nom du propriétaire du conteneur. Cela explique la grande taille du tesson par rapport au texte, comme le remarque l'éditrice.

\section{O.Edfou Copte 127 [JLF]}

L. 3: п2גM est un technonyme d'après Ch. Kuentz dans Fouilles franco-polonaises. Rapports. I. Tell Edfou 1937, Le Caire, 1937, p. 196. 


\section{O.Edfou Copte 128 [JLF]}

L. 8: on a là la première attestation papyrologique claire du mot ö $\rho \mu \alpha$, que transcrit le copte $2 \alpha \mathrm{pMd}^{22}$, avec une acception vestimentaire. L'éditrice renvoie justement à Lampe, A Patristic Greek Lexicon, qui enregistre le sens d'«habit monacal». On peut en fait ajouter deux autres occurrences papyrologiques qui avaient échappé jusqu'ici: P.Münch. III 126, 9 (IV s.?), où le mot (non accentué ni pourvu d'esprit par l'éditeur) se rencontre dans une lettre où il est question d'un

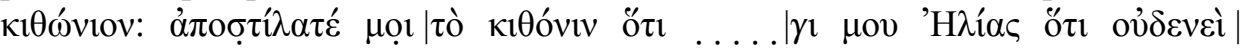

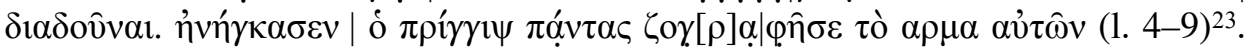

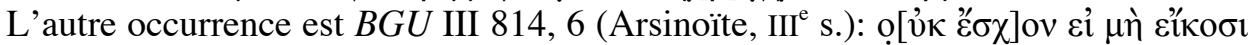

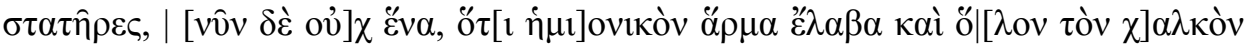

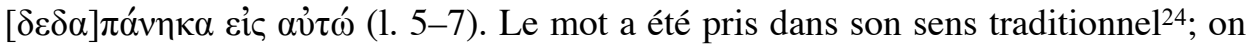
ne s'est pourtant pas étonné du prix bien bas de ce chariot (19 statères). La difficulté tombe si on lit $\chi \varepsilon 1 \mu]$ ovikòv ö $\rho \mu \alpha$ «un harma d'hiver». Pour l'emploi de $\chi \varepsilon 1 \mu \omega v i \kappa o ́ s$ appliqué à un vêtement, cf. P.Oxy. XVI 1901, 36-37 (VI s.): $\tau \hat{\omega}[v$

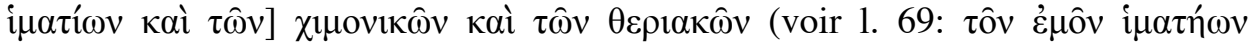

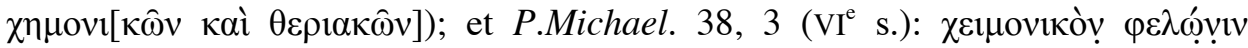

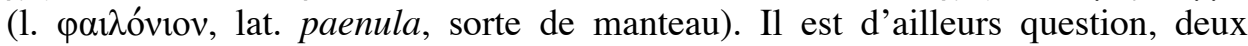

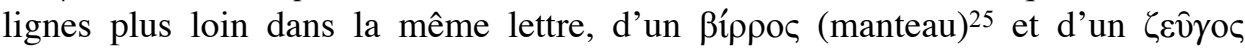
i $\mu \alpha \tau_{i}^{\prime} \omega(v)[\delta \varepsilon \rho \mu] \alpha \tau i v \omega v$ (vêtements de cuir). Il semble que ö $\rho \mu \alpha$ désigne un manteau grossier, qui, comme d'autres mots (ainsi paı入óvıov cité à l'instant), a fini par désigner un vêtement de moine.

\footnotetext{
${ }^{22}$ Le mot copte est nouveau dans les documents si l'on met de côté la liste de mots scolaires conservée par le P.Rain. Unterricht Kopt. 243, 39 (X/XI ${ }^{\mathrm{e}}$ s.): фармג.

${ }^{23}$ Les éditeurs proposent de comprendre, d'après le latin arma, «Kriegsgeräte», sans rejeter la possibilité de lire ö $\rho \alpha \alpha$ (note aux 1. 8-9).

${ }^{24}$ Cf. les traductions de W. Schubart, Ein Jahrtausend am Nil, $2^{\mathrm{e}}$ éd., Berlin, 1923, n 71 ; J.G. Winter, Life and Letters in the Papyri, Ann Arbor, 1933, p. 102-103; A.C. Johnson, An Economic Survey of Ancient Rome. II: Roman Egypt to the Reign of Diocletian, Baltimore, 1936, p. 478-479, $\mathrm{n}^{\circ} 306$.

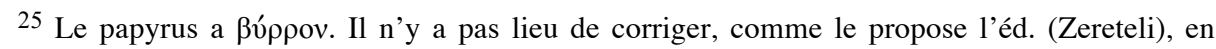

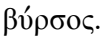




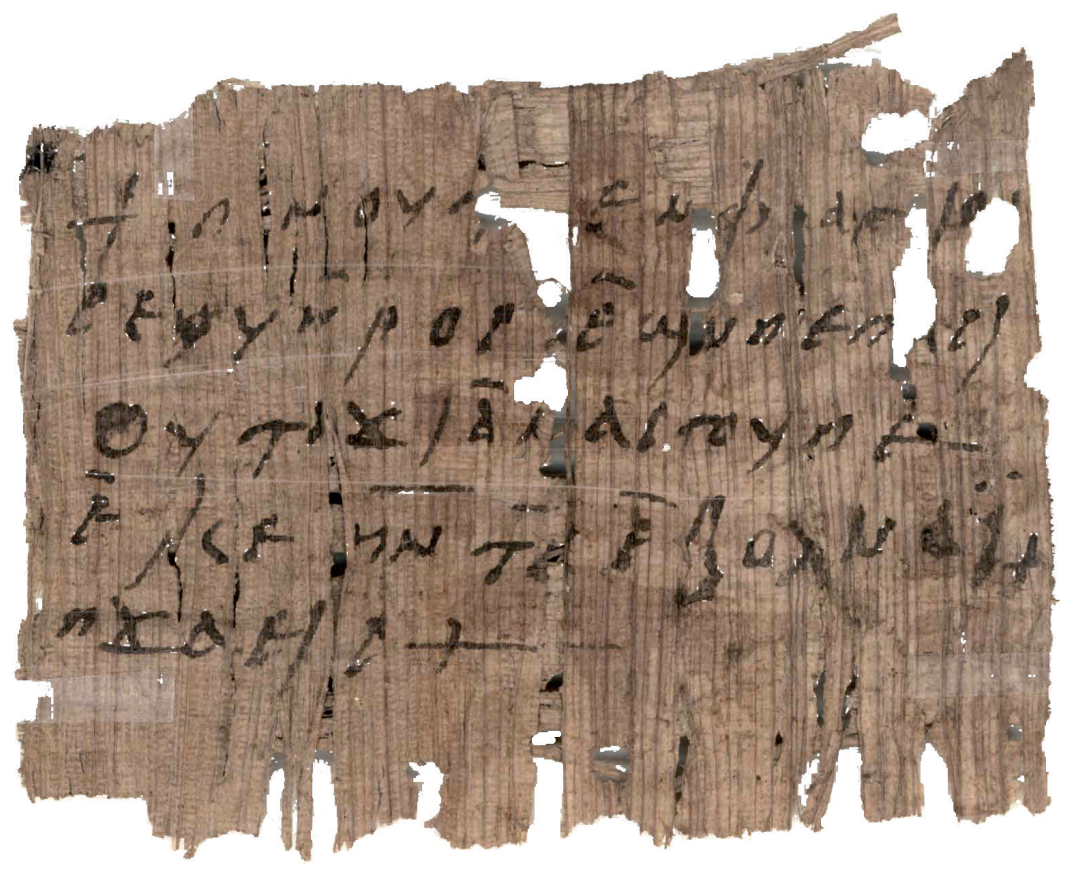

Orakel des Heiligen Severus (P.Colon. ägypt. Inv. 10211); zu: G. Schenke, S. $65 \mathrm{ff}$.

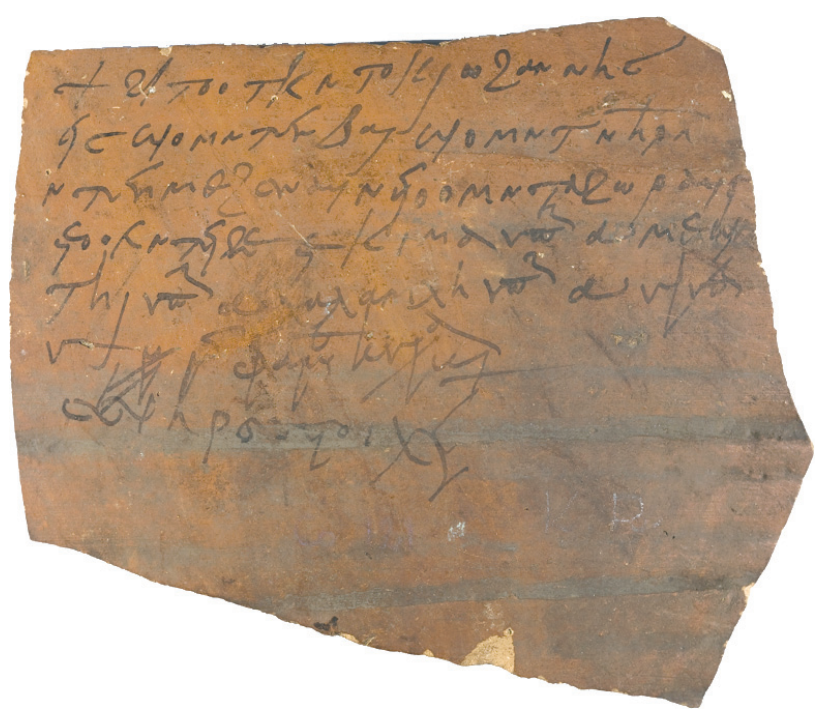

Quittung für Wein (O.Vars. Inv. 139901); zu: A. Delattre et J.-L. Fournet, S. 84. 